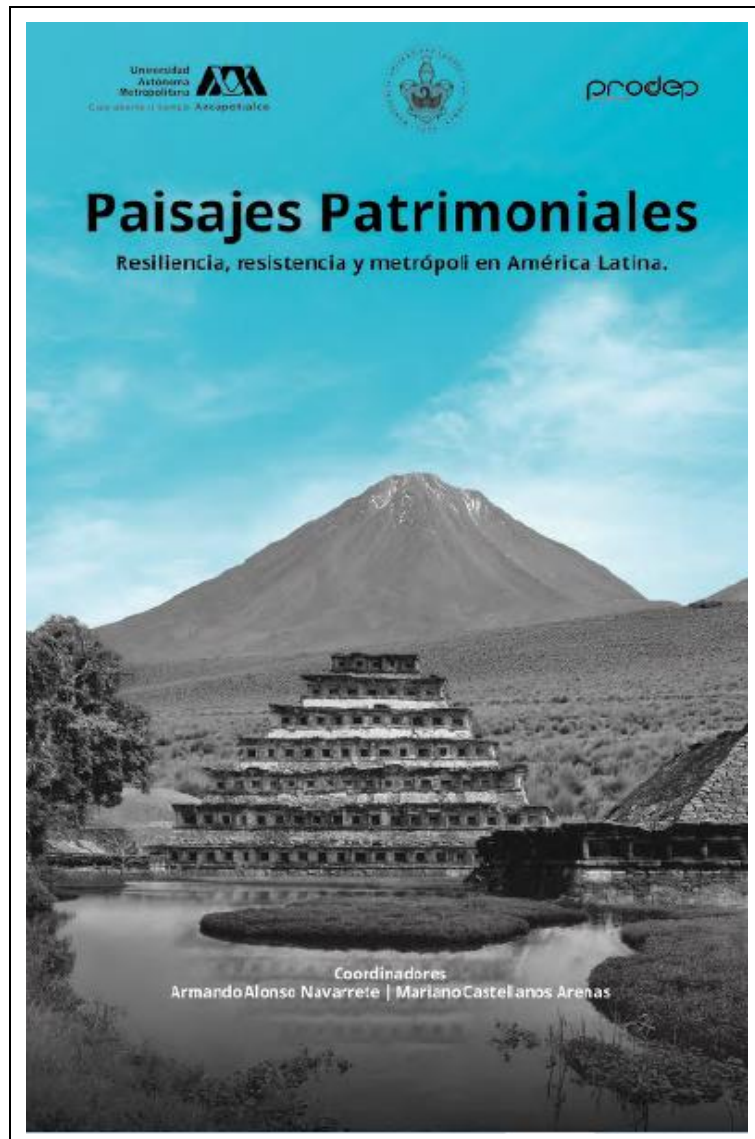

Velázquez García, Mario Alberto (2020).

ORCID: 0000-0001-9869-2188

Helene Balslev, Clausen (2020).

ORCID: 0000-0003-0148-3190

\title{
La gestión social de zonas arqueológicas y el Estado nacional. El caso mexicano.
}

p. $25-47$

En:

Paisajes patrimoniales. Resiliencia, resistencia y metrópoli en América Latina. II. Entre la teoría y el método / Armando Alonso Navarrete y Mariano Castellanos Arenas, coordinadores. Ciudad de México: Universidad Autónoma Metropolitana, Unidad Azcapotzalco, 2020.

Fuente: ISBN 978-607-28-1996-2 (versión electrónica).

Relación: http://hdl.handle.net/11191/7575

\begin{tabular}{|c|c|c|}
\hline $\begin{array}{c}\begin{array}{c}\text { Universidad } \\
\text { Autónoma } \\
\text { Metropolitana }\end{array} \\
\text { Casa abierta al tiempo Azcapotzalco } \\
\text { Universidad Autónoma Metropolitana } \\
\text { Unidad Azcapotzalco } \\
\text { https://www.azc.uam.mx/ }\end{array}$ & $\begin{array}{l}\text { Clencias v Artes para el Diseño } \\
\begin{array}{l}\text { División de Ciencias y Artes para } \\
\text { el Diseño }\end{array} \\
\text { https://www.cyad.online/uam/ }\end{array}$ & $\begin{array}{l}\text { medioambiente } \\
\text { Departamento del Medio } \\
\text { Ambiente } \\
\text { http://www.medioambiente.azc } \\
\text {.uam.mx/jefatura.html }\end{array}$ \\
\hline & $\begin{array}{c}\text { Área de Investigación } \\
\text { Arquitectura del Paisaje } \\
\text { Área de Investigación } \\
\text { Arquitectura del Paisaje }\end{array}$ & \\
\hline
\end{tabular}

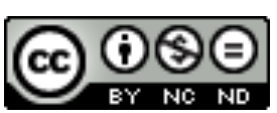

Excepto si se señala otra cosa, la licencia del ítem se describe como

Atribución-NoComercial-SinDerivadas

https://creativecommons.org/licenses/by-nc-nd/4.0/

D.R. C 2020. Universidad Autónoma Metropolitana, Unidad Azcapotzalco, División de Ciencias y Artes para el Diseño, Departamento del Medio Ambiente, Área de Investigación Arquitectura de Paisaje. Se autoriza copiar y redistribuir el material en cualquier medio o formato, siempre y cuando se den los créditos de manera adecuada, no puede hacer uso del material con propósitos comerciales, si remezcla, transforma o crea a partir del material, no podrá distribuir el material modificado. Para cualquier otro uso, se requiere autorización expresa del titular de los derechos patrimoniales. 


\title{
La gestión social de zonas arqueológicas y el Estado nacional. El caso mexicano.
}

\author{
Dr. Mario Alberto Velázquez García \\ El Colegio del Estado de Hidalgo, México. \\ mvelazquez@elcolegiodehidalgo.edu.mx \\ Dra. Helene Balslev Clausen \\ Universidad de Aalborg, Dinamarca. \\ balslev@cgs.aau.dk
}

Resumen: Este trabajo busca analizar cuál ha sido el papel estatal en construir y definir el patrimonio cultural en México. El análisis sociológico sobre la relación de los estados con la conservación del patrimonio es un tema poco explorado en los estudios patrimoniales. Este trabajo busca contribuir a este tipo de estudios. Analizamos la relación que existe entre la misma edificación del Estado y los mecanismos para la conservación del patrimonio y las zonas arqueológicas.

Palabras clave: Estado, zonas arqueológicas, profetas del pasado, construcción de la realidad y marco jurídico.

\begin{abstract}
The paper seeks to analyze the state's role in constructing and defining cultural heritage in Mexico. There is very scarce research done on the relations between patrimonial conservation and the state from a sociological perspective. We seek to close this gap in research by analyzing relations between the Mexican state construction and mechanism for heritage conservation and archaeological sites.
\end{abstract}

Keywords: State, archaeological zones, prophets of the past, construction of reality and legal framework.

\section{Introducción.}

A cuatro kilómetros de Tulancingo se encuentra la población de Huapalcalco. Por la localidad cruzaba el río Huapalcalco entre el Cerro de la Mesa y Cerro de Huiztli, que se acumulaba conformando una laguna con el mismo nombre del pueblo. Tanto el río como la laguna actualmente se encuentran secos. Aquí se localiza un conjunto de ruinas prehispánicas. Estas construcciones tenían, muy probablemente, una función de templos adoratorios, pero también fueron una especie de residencia de descanso para algún gobernante. No existe todavía una investigación que determine con exactitud quién fue el dirigente que decidió construir este conjunto, pero está dedicado a "Quetzalcoatl", personaje que está entre el mito y la realidad. La vista desde estas construcciones prehispánicas hacia el Valle de Tulancingo y el próximo lago de Huapalcalco tuvo que 
ser majestuosa: a espaldas los cerros, un río atravesando y un espejo de agua. A 120 kilómetros en línea recta desde este lugar se encuentra Teotihuacán, la "ciudad de los dioses". En la actualidad, las construcciones prehispánicas que permanecen, -pequeñas en tamaño si las comparamos con otras zonas arqueológicas-, no han sido objeto de mucho interés por el Estado mexicano y los arqueólogos. Las construcciones de Huapalcalco se encuentran en su gran mayoría semi- enterradas e invadidas por el pasto. Poco a poco las casas de los actuales habitantes se ubicaron a su alrededor; algunas de las estructuras arqueológicas están en el patio trasero de una vivienda y son usadas como zona de pastaje de vacas. Circulaban historias sobre la venta de algunos objetos encontrados en las ruinas o las cuevas aledañas.

El Estado federal mexicano, el Instituto Nacional de Antropología e Historia (INAH), el gobierno del Estado de Hidalgo y el Municipio de Huapalcalco no han mostrado tener un especial interés por conservar, investigar y difundir este conjunto específico de ruinas prehispánicas. ¿Cuáles son las razones que explican esta falta de interés? ¿Por qué un Estado y sus instituciones deciden ignorar un espacio histórico y en cambio apoyar otros? ¿Por qué no es importante Huapalcalco (así como otros lugares donde existe restos arqueológicos), para el Estado federal mexicano a pesar de que la población local ha buscado preservar este patrimonio? Para poder responder a este tipo de preguntas es necesario analizar, entre otros temas, cuál es la gestión del Estado mexicano respecto a las zonas arqueológicas.

De forma general, podemos definir la gestión como una actividad técnica realizada por cualquier organización en la búsqueda por resolver un problema, lograr un objetivo o concretar un proyecto. Dicho en otras palabras, es la planeación de los medios que serán utilizados por un grupo para lograr un objetivo. Decimos que se trata de una tarea técnica, porque al usar el término de "gestión" nos enfocamos en la creación e implementación de estrategias que optimicen los resultados que puede obtener una organización.

En este escrito partimos de la siguiente premisa: la gestión de las zonas arqueológicas en México es una actividad con múltiples dimensiones. La construcción del mismo Estado mexicano ocupa un papel central, pero también son relevantes los aspectos económicos, legales, culturales, la interacción entre los distintos grupos involucrados, los usos (locales, gubernamentales y comerciales) de la zona y la política. Entre otras cosas, esto significa que la gestión de nuestro patrimonio arqueológico no es (únicamente) un tema técnico, histórico o de políticas públicas sino está relacionado conla misma definición de los límites y alcances de lo 
estatal. En este sentido, para poder entender lo que ha pasado en zonas arqueológicas específicas como Huapalcalco es necesario analizar el proceso de "construcción estatal de la realidad". Esto significa retomar la postura sociológica de Durkheim quien postuló que una de las funciones centrales de los Estados modernos era la "integración lógica", es decir, generar una serie de definiciones y marcos comunes que permitieran las diversas interacciones dentro de una sociedad; esto abarca desde un idioma, hasta el uso de un sistema de medidas y principios de colaboración en espacios públicos (Durkheim, 2013). La gestión de las zonas arqueológicas es uno de los elementos mediante los cuales el Estado mexicano da integración lógica al pasado que lo justifica. En un primer apartado analizaremos el papel que tiene el Estado en la definición del patrimonio y su gestión.

\section{El monopolio estatal en la definición del pasado.}

Entre los trabajos pioneros de sociología, los escritos de Max Weber fueron los primeros que intentaron explicar el rol del Estado como el principal agente del orden social. Para este autor, el Estado es una asociación política que se caracteriza por contar con un aparato burocrático y una legitimidad basada en un conjunto de principios y normas generales (las leyes) y la racionalidad de su aparato administrativo.

Una organización gobernante será llamada 'política' en la medida en que su existencia y orden sean continuamente salvaguardados dentro de un territorio determinado por la amenaza de la aplicación de la fuerza física por parte de un órgano administrativo. Una organización política coercitiva con operación continua sea llamada "Estado" en la medida que su órgano administrativo ostente para sí el monopolio legítimo de la violencia física para proteger su orden (Weber, (1919) 1984: 54).

Para Weber las asociaciones deben ser estudiadas por los medios que utilizan más que por los fines que persiguen. En este sentido, el autor planteó que la característica de los Estados modernos es haber generado un monopolio en el uso de la fuerza dentro de sus territorios. Esto no significa que este sea el único recurso de estas asociaciones para lograr la dominación, por el contrario, se trata del último recurso.

Durkheim en su estudio sobre la sociedad francesa le otorgó un papel axial al Estado considerándolo como uno de los principales agentes en el proceso de incorporación de los sujetos a una sociedad determinada (socialización). Entre las principales funciones del Estado en las sociedades modernas, este autor propuso la "integración lógica", es decir, el generar una serie de definiciones y marcos comunes que permiten las interacciones entre los individuos y grupos; esta serie de intervenciones 
estatales comprendían desde la enseñanza de un idioma común y oficial, hasta el uso de un sistema de medidas y principios de colaboración en espacios públicos (Durkheim, 2013). Por su parte, para Weber el Estado es una forma de dominación política que busca ejercer el poder sobre un grupo de población dentro de un territorio determinado, para ello es necesario que consolide legitimidad sobre las razones que le confieren este atributo particular (Weber, 1984). Mientras, Marx ubicó al Estado dentro del sistema capitalista como un instrumento que le permitía a una clase el control de los medios de producción por medio de un aparato jurídico y administrativo (Marx, 2004).

Tres autores que propusieron nuevos enfoques para analizar el Estado fueron Michel Foucault, Pierre Bourdieu y Norbert Elías. Ellos abandonaron el intento por generar una definición macro-sociológica respecto con la composición y funciones de todos los Estados, e incluso, sobre qué es el Estado. A pesar de partir de posturas distintas, los tres coincidieron en señalar que el Estado tenía características centrales que no fueron exploradas en la definición de Weber (Foucault, 2004; Bourdieu; 2014; Elías, 2016; Weber, 1984).

Estado es aquella comunidad humana que, dentro de un determinado territorio, reclama (con éxito) para sí el monopolio de la violencia física legítima. Lo específico de nuestro tiempo es que a todas las demás asociaciones e individuos sólo se les concede el derecho a la violencia física en la medida en que el Estado lo permite. El Estado es la única fuente del "derecho" a la violencia (Weber, 2009: 83-84).

Bourdieu propuso una adición a la definición de Weber del Estado como el poseedor no sólo del monopolio de la violencia sino de la construcción de la representación legítima del mundo social, es decir el Estado tiene una dimensión simbólica. Esta última es perceptible a través de la acción directa de las instituciones y sus funcionarios (Bourdieu, 2014: 243). Desde la perspectiva de Bourdieu el Estado es, en sí mismo, uno de los componentes generales de la estructura de las sociedades modernas, dado que su funcionamiento es el que produce las categorías con las cuales son definidas las mismas categorías que permiten definir los distintos componentes que componen a una sociedad (Bourdieu, 2014: 230).

Entonces, el Estado funciona no sólo por la violencia, sino a partir de una acumulación originaria de capital simbólico, que le permite imponer un conjunto de ideas sobre el cómo deben ser nombrados los objetos y cuáles son las reglas básicas de comportamiento dentro de una sociedad. 
El Estado está en un estado (por así decirlo) de imponer de manera universal, a escala de cierta extensión territorial, unos principios de visión y división, formas simbólicas, principios de clasificación, lo que a menudo llamo un nomos, recordando la etimología propuesta por Benveniste, según la cual nomos viene de nemo, "compartir", "dividir", "compartir partes separadas" por una especie de diachrisis, como decían los griegos, de "división originaria" (Bourdieu, 2014: 231).

En Bourdieu el Estado es el principal agente en la construcción de la realidad, incluso más allá: es quien erige las categorías, definiciones, principios y metas que nos permiten vivir en las sociedades modernas. En este sentido, el papel estatal en el tema del orden social rebaza por mucho el control y la dominación económica para abarcar un tema simbólico: el Estado el integrador social de las estructuras cognitivas y evaluativas (Bourdieu, 2014: 236).

Pierre Bourdieu retomó el término Weberiano de "profetas éticos" o "profetas jurídicos" para concretizar su propuesta de análisis a partir del concepto de historia genética. El objetivo de este autor era analizar la formación del discurso estatal y el desarrollo de la labor de aquellas personas que serían sus depositarios: los funcionarios. El objetivo de Bourdieu era observar las prácticas concretas que permiten la reproducción de un cierto orden social; cuáles eran los mecanismos concretos con los que opera un Estado más allá de las reglas generales de la burocracia de Weber. Para ello propuso analizar a los individuos que en su cotidianeidad hablan, trabajan y construyen la sociedad en nombre del Estado: los funcionarios.

Bourdieu partió de hacerse la siguiente pregunta: ¿Cómo es posible que un conjunto de individuos se abrogue la facultad de hablar como los dueños de un saber particular? Para este autor, este proceso inicia en la misma construcción histórica de los Estados modernos cuando estos comenzaron a acumular los distintos capitales (económico, cultural, social y simbólico) y con ello, la legitimidad sobre el uso y la distribución de estos.

Los profetas del pasado operan bajo un conjunto de normas y reglamentos que delimitan su actuación; estos son los límites y alcances de las capacidades estatales que les son conferidas. Para Bourdieu una de las características principales de estos (u otros) grupos de burócratas es la especialización que logran en el manejo de los distintos reglamentos y procedimientos estatales, no en la materia misma sobre la que versa su trabajo (en este caso en la preservación de objetos relacionados importantes para la historia de México), sino en los reglamentos que regulan los trámites relacionados. 
Los funcionarios y los ciudadanos (clientes) entran en interacción cargados de una serie de recursos (capital económico, cultural y social) que les permiten buscar influir en el resultado. El uso de estos medios está determinado por los intereses y las finalidades que cada uno de los actores tiene. Esto tiene importancia particularmente en las interacciones donde se busca influir en la definición y uso de bienes públicos; distintos actores buscan afectar en los distintos momentos de creación, operación y diagnóstico de las acciones de gobierno que operativizan el uso de los bienes públicos (Etzioni, 1967).

Como fue señalado desde los estudios de Weber, la burocracia funciona a partir de una jerarquía que genera un orden de toma de decisiones y de facultades entre los distintos empleados. Esta jerarquía reproduce no sólo un control interno sino también permite construir filtros hacia afuera; no todos los solicitantes de atención pueden alcanzar todas las esferas de atención, ni son atendidos por las mismas oficinas. Uno de los temas relacionados a la jerarquía e interacción de las burocracias son sus límites: ¿Dónde está la frontera entre los distintos grupos? (Bourdieu, 2014: 33). Es decir, cuáles son los grupos con los que la interacción de estos funcionarios se vuelve cotidiana e interconectada. En algunos casos, la interacción entre organizaciones es tan significativa que resulta difícil establecer donde termina el objetivo e interés de cada organismo. En el caso de los profetas del pasado, uno de los grupos con los que mantienen una interacción más intensa son los centros de investigación (universidades, colegios de profesionistas, entre otras). La interacción de los investigadores con los profetas del pasado requiere ser investigada.

Aunque el Estado no fue el tema central de Norbert Elías, sus trabajos sobre la vida cotidiana proponen una vía distinta para analizar el papel de Estado en las sociedades contemporáneas. Uno de los puntos centrales en la investigación de Norbert Elías fue el proceso de construcción de la civilización. Para Norbert Elías este fue un mecanismo por medio del cual el Occidente se auto- diferenció de las distintas culturas y su propio pasado. La palabra civilización es un aparato de afirmación de "superioridad":

El concepto resume todo aquello que la sociedad occidental de los últimos dos o tres siglos cree llevar de ventaja a las sociedades interiores o a las contemporáneas más "primitivas". Con el término de "civilización" trata la sociedad occidental de caracterizar aquello que expresa su peculiaridad y de lo que se siente orgullosa: el grado alcanzado por su técnica, sus modales, el desarrollo de sus conocimientos científicos, su concepción del mundo y muchas otras cosas... (Elías, 2016: 83). 
Elías escogió analizar la construcción de la idea de civilización a partir de aquellos escritos que tenían como objeto instruir a las personas sobre las prácticas que permitían ejercer un autocontrol en el "decoro externo del cuerpo", particularmente, los manuales sobre buenas costumbres. Uno de los escritos utilizados por el autor para su análisis son las obras de Desiderius Erasmo, también conocido como Erasmo de Róterdam (Elías, 2016, 132). En este trabajo, Elías mostró el papel que desempeñó el desarrollo de las distintas instituciones estatales en la construcción de un "monopolio de la violencia"; los Estados generan una serie de normas, prácticas y reglamentos que fomentaron el auto- control de distinto tipo de emociones y pasiones, o en su caso su castigo por medio de penas legales. En este sentido, el trabajo de Elías complejiza la propuesta de Weber sobre el control de la violencia y el Estado para mostrar que este dominio abarcaba muchos más ámbitos que el control policial, fuerza física, grupos armados y las guerras; los Estados modernos fueron coparticipes en la creación de una red funcional de control de los comportamientos, las emociones y las interacciones dentro de las nuevas sociedades europeas.

Para Elías la continua interconexión entre los miembros de una sociedad genera el orden social, pero este no es un resultado que pudiera haber sido planeado por un solo sujeto; la acción conjunta e interrelacionada de los individuos puede generar resultados y transformaciones no esperadas. En este sentido, el orden social no pueda seguir una línea prestablecida o incluso postular la evolución como eje central; las sociedades son un resultado no predecible de la interdependencia de los individuos, las instituciones del orden social que se acumulan históricamente y las transformaciones que los sujetos introducen en sus acciones interconectadas. Esta postura desliga a los procesos sociales de una "evolución" constante, pues como sucede en realidad, las civilizaciones desaparecen o son absorbidas por otras: ni los Estados modernos son el "fin de la historia" ni tampoco es el destino ineludible del capitalismo el socialismo; la transformación es un proceso que pude generar mayor orden social o destruirlo.

Por su parte el trabajo de Benedict Anderson en su libro Comunidades Imaginadas significó una propuesta innovadora en términos teóricos y metodológicos para el estudio sobre el Estado. Al igual que Norbert Elías, Anderson desmontó la idea de un Estado cristalizado y monolítico construido de una vez, para mostrar que se trataba de un conjunto de prácticas, normas e instituciones que se iban construyendo y negociando a lo largo del tiempo y del territorio. En este sentido, el Estado no es el resultado de un proyecto terminando y definitivo de una persona o grupo, sino un conjunto de prácticas, intereses y conflictos que van transformándose, y con ello cambiando al mismo Estado y el contenido de sus componentes. En lo metodológico, su trabajo permitió ampliar la idea Durkheniana del orden 
social para centrar el estudio del Estado, no en sus definiciones ideológicas, sus grandes personajes o las leyes generales, para centrarse en las prácticas y elementos cotidianos que las instituciones estatales proponían y buscaban imponer en la cotidianeidad (Anderson, (1983) 1993).

\section{Las tecnologías estatales para el monopolio sobre el pasado.}

A diferencia de la concepción Weberiana sobre la burocracia, Bourdieu y Foucault plantearon que las tecnologías de los expertos estatales no están destinadas únicamente a producir un funcionamiento racional (finesmedios) de las organizaciones, sino también buscan mantener el control; es decir, establecer los límites entre los miembros del grupo y el resto de la población. Las organizaciones buscan regular el acceso de las personas para obtener los beneficios o resultados que son la meta explicita del grupo. Para analizar esta parte, retomaremos como un complemento a la propuesta de Bourdieu el esquema analítico de Foucault sobre las prácticas concretas mediante las cuales se ejerce el poder. Esto es necesario porque la propuesta de Bourdieu no generó un aparato mico- analítico específico sobre las tecnologías cotidianas utilizadas para el diseño y la operación de las políticas públicas. Por su parte la propuesta de Foucault y particularmente la aplicación de ella propuesta por Dean, nos permitirá contar con un esquema que permita diseccionar los distintos componentes de las prácticas de los funcionarios estatales.

Tanto para Bourdieu como para Foucault, una de las funciones centrales de los Estados modernos es la producción de la verdad, pero no cualquier tipo de verdad sino aquella relativa a su propia identidad o funcionamiento (Foucault 2004; Bourdieu, 2014). La verdad estatal que se busca producir es la de aquellos elementos que están bajo su control: los individuos, la población y el territorio. A este conjunto de tecnologías destinadas a la producción de la verdad, Foucault las nombró formas de normalización haciendo referencia a los procedimientos estatales utilizados para enfrentar y conocer los fenómenos, el lograr determinar cuál es su funcionamiento regular normal, o bien, los diferentes grados con los que se presenta. La normalización de un acontecimiento se construye por medio de su identificación, cuantificación y racionalización en sus incidencias individuales, éstas, como parte de un colectivo de sucesos similares. Esta construcción del caso particular requiere entonces de la medición del hecho o acontecimiento (Foucault 2004).

El uso de las tecnologías está determinado por un conjunto de principios que dan lógica al funcionamiento de cada una de las organizaciones estatales. El primero es la visión que los mismos integrantes (en este caso los funcionarios) tienen sobre su propia acción dentro de la organización: sus actos son considerados como más general y con mayores beneficios 
"públicos", a comparación de los de una persona particular. Esta visión de su operación es aplicada al interior de las mismas estructuras de gobierno de tal manera que los agentes del gobierno estatal se mirarán a sí mismos más incluyentes en su actuación que las de los órganos locales. Un segundo principio está relacionado con la manera en que las instancias oficiales seleccionan a las personas que serán involucradas en las distintas etapas de un tema público; serán seleccionados "los agentes pertinentes porque tienen algo que ver con este problema y algo pueden en este problema; tienen el poder específico que permite ser eficiente, producir efectos" (Bourdieu, 2014: 34). El tercer principio es seleccionar a los agentes porque estos poseen características, conocimientos, capitales (simbólicos, culturales e incluso económicos) que son consideradas como pertinentes para poder actuar de una manera eficiente en el tema específico.

Algunas de las herramientas concretas utilizadas para este fin son los censos, que desde tiempos del imperio romano le han permitido a un Estado conocer el número, las características y la composición de las personas, objetos y lugares que se encuentran dentro de su territorio. El perfeccionamiento de los instrumentos y las técnicas censales sin duda han ido ampliando las funciones estatales que son posibles; no sólo recaudar impuestos, sino planear y proyectar el gasto gubernamental, generar medidas de control sobre distintos fenómenos, etcétera.

Los funcionarios (en nuestro caso los "profetas éticos" del pasado) son los sujetos que ejercen un papel central en el uso de estas herramientas estatales de producción de verdad. Para poder ocupar este rol, es necesario que cada uno de estos sujetos atraviese por un proceso de transformación que le confiera conocimiento, experiencia y certificación estatal. Tradicionalmente la educación, -certificada y validada por el mismo Estado, - y el trabajo (en entes privados o públicos) han sido las dos fuentes que cumplen este rite de passage que genera a los profetas éticos. Por supuesto estas no son las únicas vías que permiten a un sujeto convertirse en un experto, en casos particulares estos pueden ser heredados o transmitidos por el contacto continuo de la persona con alguien que fue el mismo un profeta ético y que le "transmitió" su conocimiento.

Una de las tecnologías utilizadas en distintas instancias del Estado mexicano es lo que nosotros denominaremos como la "muralla de papel" gubernamental. Por esta hacemos referencia a una compleja red de procedimientos jurídicos, trámites y oficinas burocráticas encargadas de atender y dilatar, desde el centro de toma de decisiones, una decisión gubernamental que pueda ser considerada como (temporalmente) perjudicial para los tomadores de decisiones (Velázquez Guzmán, 1982; Velázquez, 2016). 
La muralla de papel significa la generación de una serie de trámites que en términos prácticos retrasan la respuesta de las instituciones ante peticiones hechas por agentes externos. Estos trámites son justificados como parte de los procesos internos que le permitan a la organización iniciar y dar seguimiento a cualquier petición, pero también significan la generación de atributos y capacidades exclusivas para otras instancias internas de la propia organización o de otras agencias estatales o que cuentan con el aval de este.

\section{El Estado como productor de la verdad.}

Para Foucault el ejercicio del poder estatal tenía en la producción de la verdad uno de sus objetivos principales; tanto en lo que refiere a los individuos como a la población y el territorio (Foucault, 2004). Para este autor el Estado no fue un tema de interés en sí, sino la gubernamentalidad es decir, un conjunto de instituciones sociales que concentra una red de prácticas y técnicas del ejercicio del poder en un periodo determinado de la sociedad (Foucault, 2000).

Dean (1999) utilizó los trabajos de Foucault como base para proponer cuatro principios generales con los cuales las instituciones gubernamentales organizan sus prácticas generales frente a los gobernados:

1. Formas características de visibilidad y maneras de percibir. Todas las agencias de gobierno hacen una selección sobre los problemas, grupos, objetivos que son seleccionados, visibles para el Estado. Esto implica, entre otras cosas, que un conjunto de comportamientos y prácticas (tanto del gobierno como de las personas) serán consideradas benéficas y otras serán vistas como rivales a los objetivos de la agencia (Dean, 1999; Schneider Ingram, 1993).

El Estado realiza una clasificación sobre cada uno de los individuos y sus comportamientos. Esta clasificación ordena y selecciona cuáles son las facetas de una conducta o los grupos que serán atendidos por las distintas organizaciones estatales.

En nuestro estudio, esto se refiere a los criterios implícitos y explícitos con los que el Estado mexicano ha decidido cuáles son las zonas arqueológicas que requieren ser conservadas y cuáles no, así como aquellas personas o grupos sociales que pueden participar en estas actividades de exploración, conservación y exhibición de monumentos históricos.

2. Formas de producir el conocimiento. Los Estados requieren de la producción y el control del flujo de información sobre los distintos elementos que interactúan en su espacio de poder: grupos sociales y sus características, formas de intercambio, recursos naturales y 
sociales, límites y perspectivas (Giddens, 1985). Esta medición implica generar un conjunto de definiciones sobre los sujetos, grupos o comportamientos lo que implica la institucionalización de prácticas para recabar la información, pero también la normalización de una escala de valores.

La denominación de zonas arqueológicas genera una distinción entre espacios que son reconocidos como poseedores de elementos prehispánicos de valor, pero al mismo tiempo, se genera una distinción frente a otros lugares que no han obtenido esta denominación a pesar de contener ruinas de la misma época histórica. Los primeros reciben un tratamiento legal y en recursos estatales distinto.

3. Formas de racionalidad. Los Estados generan un conjunto de formas de comportamiento y de dirección (de los comportamientos) desde sus agencias. Estas constituyen la "integración lógica" de Durkheim; es decir los comportamientos que serán enseñados y aceptados como un calendario, horario, idioma, sistemas de medida, leyes etcétera (Dean, 1999; Durkheim, 2013).

El Estado determina principios generales que permiten y regulan muchos aspectos de las interacciones humanas. Los Estados van determinando desde las reglas del idioma que hablamos, el calendario y el tiempo (reloj) con el que programamos nuestras actividades. Los Estados son los encargados de promulgar las reglas generales que deben de seguir una serie de comportamientos socialmente normalizados como los doctores, abogados, profesores, etcétera.

El Estado requiere definir un conjunto de metas que serán alcanzadas mediante el funcionamiento de sus agencias. Algunas de estas metas tienen un carácter general y abstracto como la libertad de los ciudadanos, la salud, el bienestar, etcétera. También es necesario que se presenten metas específicas para cada una de las agencias gubernamentales como reducción de las muertes por cáncer de próstata, aumento en el número de empleos, etcétera.

Para alcanzar este conjunto de metas los Estados requieren generar un conjunto de estrategias específicas que serán operados directamente por las agencias de gobierno o podrán ser el resultado de la actividad de otros grupos o la cooperación entre sectores sociales con el gobierno.

4. Formación de sujetos. Los Estados generan una definición sobre las características que tiene los distintos grupos y sujetos que habitan o interactúan dentro de su territorio. Ante cada uno de estos agregados o individuos particulares, los Estados desplegaran un conjunto diferenciado de formas de medición, intervención, 
vigilancia, redireccionamiento de su actividad, impulso o incluso prohibición de su actuar.

El Estado, en este caso el mexicano, generó distinciones binarias básicas entre autorizados/no autorizados, expertos/no expertos, respecto con la definición de qué lugares, objetos, historias y demás objetos materiales e inmateriales serían considerados como parte de la historia oficialmente validada de México.

\section{El indígena y el mestizo en la construcción del nuevo sujeto del Estado mexicano.}

México libró una guerra civil interna durante las primeras dos décadas del siglo XX y no comenzaría su desconsolidación hasta 1921, con un acuerdo entre las facciones militares que triunfaron. Posteriormente al término del conflicto armado, el Estado mexicano tenía varias tareas centrales para consolidar su autoridad: la pacificación del país, la reconstrucción de la identidad nacional, el control en la explotación de los recursos materiales y las actividades productivas que tenían lugar dentro de su territorio, así como generar un conjunto de políticas públicas que se aplicaran en todos los niveles de gobierno (Mallon, 1994).

Al mismo tiempo, el Estado mexicano utilizó distintos mecanismos en su búsqueda por (re)construir un discurso cultural que le permitiera alcanzar una cierta unidad nacional. Para ello resultó fundamental seleccionar un conjunto de referentes históricos, simbólicos y lingüísticos que identificaran a las distintas zonas que componían el territorio nacional con un proyecto común (Rockwell, 1994). Un elemento central utilizado por los gobiernos mexicanos en la conformación de la narrativa estatal sobre México fue el pasado prehispánico y las culturas indígenas (Cosío Villegas et al, 1974). El discurso nacional reflejaba la definición del nuevo grupo en el poder sobre la composición cultural y política de México. Antes de la Revolución, el país no era una nación, sino un conjunto de regiones con expresiones culturales, sociales y económicas distintas. La pacificación de México sólo sería posible bajo la consolidación de un ideal común (Joseph and Nugent, 1994; Mallon, 1994).

La reconstrucción del discurso cultural estatal implicó, en términos de políticas públicas, la fabricación de un agente que encarnara los distintos elementos simbólicos, históricos y políticos que habían sido seleccionados: este ente social fue el mestizo, que sería el eje central de la unificación cultural nacional, pero también el principal beneficiario de las acciones de política del Estado mexicano. Agentes culturales como los españoles y en general los extranjeros serían considerados oponentes al proyecto estatal para lograr el monopolio en la creación de la cultura mexicana. 
Como lo argumenta Guillermo Bonfil Batalla, el proyecto cultural del Estado mexicano post revolucionario buscaba utilizar símbolos de su pasado, como la época prehispánica, pero mantenía su mirada en lo que era considerado como el futuro: la modernización occidental (Bonfil, 1990). Este plan cultural tenía una clara expresión en las ocupaciones económicas que serían apoyadas mediante las políticas públicas. Aquellas actividades productivas que permitieran la modernización industrial serían apuntaladas y financiadas, mientras aquellas que significaran la continuidad de formas productivas tradicionales serían ignoradas o sancionadas.

En el proyecto estatal, la cultura indígena -la presente y viva, no la enterrada en las pirámides- simbolizaba el pasado al que el nuevo Estado no quería regresar. Como lo muestra el trabajo de Andrés Oseguera, los rituales indígenas fueron calificados, por el gobierno porfirista y los primeros gobiernos postrevolucionarios, obstáculos que impedían el desarrollo de México (Oseguera, 2008). Dentro del esquema analítico que proponemos, los indígenas constituyeron un oponente para las primeras políticas culturales de los gobiernos post- revolucionarios.

En este sentido, la construcción social de las políticas estatales retomó los prejuicios raciales heredados de la colonia que iban dirigidos contra los indígenas. Aunque el Estado mexicano le otorgaba a este grupo un lugar privilegiado en la historia y la cultura nacional, esta posición era en calidad de culturas muertas, no presentes. Los descendientes de las culturas indígenas originarias deberían incorporarse a la nueva comunidad imaginaria constituida por los mestizos: "México era un país mestizo y los remanentes que no lo fueran debían integrarse cuanto antes" (Bonfil, 1990: 164).

Los indígenas serían destinatarios de políticas públicas que los obligaran a la unificación con el resto de la población; el idioma español fue instituido como la lengua única para la enseñanza en las escuelas de todo el territorio mexicano (Cárdenas y Arellano, 2004); igualmente, todos los trámites y documentos oficiales sólo serían redactados en español. Por su parte, los empresarios y obreros fueron integrados a un modelo corporativo de apoyos para continuar desarrollando su labor en tanto pilares de la buscada modernización (López Villafañe, 1986).

Como dijimos anteriormente, las culturas indígenas no fueron consideradas en un primer momento dentro de la narrativa de la identidad mexicana. Sin embargo, la investigación antropológica de los años treinta y cuarenta del siglo pasado, los distintos movimientos campesinos e indigenistas y la creciente presión de organizaciones y agencias internacionales para la protección de las comunidades indígenas, fueron transformando la perspectiva estatal mexicana respecto con 
los grupos originarios de México (Iturralde, 1991). Particularmente, el trabajo de antropólogos como el norteamericano Robert Redfield (1930), contribuyeron a la revaloración de las prácticas y rituales indígenas, por constituir mecanismos que favorecían a la estabilidad de las comunidades. Las poblaciones indígenas visitadas por este autor se tornaron en sitios de interés para investigadores extranjeros y nacionales. El trabajo pionero de Redfield generó un conjunto de estudios que comenzaron a crear una nueva perspectiva respecto con las prácticas culturales indígenas (Oseguera, 2008).

Desde la década de los treinta del siglo pasado, las culturas indígenas mexicanas se convirtieron en uno de los objetos de investigación recurrentes de los antropólogos norteamericanos. El que enfaticemos los trabajos hechos por extranjeros sobre las culturas indígenas mexicanas no busca menospreciar la labor de la naciente antropología mexicana dentro de la cual se produjeron sin lugar a dudas algunos de los trabajos más importantes para comprender a las culturas originarias del país-, el objetivo únicamente es señalar que dadas las características políticas del Estado mexicano, la presión externa tuvo un peso significativo en la conformación de las políticas públicas respecto con la población indígena. Las investigaciones de los antropólogos extranjeros contenían críticas directas e indirectas sobre la pobreza y exclusión que padecían estos grupos sociales. Algunos trabajos fueron los de Wendell Bennett y Robert Zingg, The Tarahumara, an Indian tribe of northern Mexico (1935); Carl Sofus Lumholtz, El México desconocido. Cinco años de exploración entre las tribus de la Sierra Madre Occidental; en la tierra caliente de Tepic y Jalisco, y entre los Tarascos de Michoacán (1945); Van Zantwijk, Los servidores de los santos. La identidad social y cultural de una comunidad tarasca (1974); Marvin Harris, Patterns of race in the Americas (1964); Frank Cancian, Economía y prestigio en una comunidad maya. El sistema religioso de cargos en Zinacantan (1976), por nombrar algunos (Oseguera, 2008).

Posteriormente, en las últimas cuatro décadas del siglo XX, los indígenas comenzaron procesos paralelos de transformación que los fueron convirtiendo en nuevos sujetos sociales, dejando de estar envueltos en un proceso de extinción para convertirse en actores políticos sobresalientes. Estas transformaciones fueron: 1) cambio en su presencia territorial, pasando de ocupar lugares marginales para insertarse dentro de las distintas ciudades y regiones; 2) el desarrollo de nuevas y más complejas formas de organización; 3) las demandas de los grupos indígenas tomaron una dimensión política y nacional sin precedente; 4) los indígenas comenzaron a generar nuevas formas de inserción dentro de la economía nacional; y 5) un nuevo patrón de relaciones entre los grupos indígenas con el Estado (Iturralde, 1991). 
Como dijimos, el pasado prehispánico comenzó a convertirse en un tema de política y de inclusión en su narrativa. Empezando por los expertos en estos temas, pero también por visitantes que deseaban conocer de primera mano las pirámides o esculturas (Villalobos, 2011). La revaloración histórica y económica de las culturas originarias es retomada por el Estado mexicano. El nuevo papel de los indígenas como beneficiarios en las políticas públicas y el sustento de la nueva legislación han significado un cambio de la relación de estos grupos sociales con algunos de los agentes que tienen un papel importante en la construcción de los lugares turísticos, como los antropólogos o las autoridades encargadas de administrar las zonas arqueológicas o museos. Las poblaciones indígenas comenzaron a utilizar el interés generado por las zonas arqueológicas o las culturas indígenas como un instrumento de empoderamiento frente al Estado o los turistas (Díaz-Andreu, 2014).

Un momento fundamental en la transformación de la posición de los indígenas en las políticas públicas en México fue el levantamiento armado del Ejercito Zapatista de Liberación Nacional en 1994. Este grupo se convirtió en un actor nacional e internacional por su explícita oposición al nuevo orden mundial (Castells, 2009). El zapatismo produjo una transformación en las estrategias gubernamentales para tratar con los grupos guerrilleros, así como en la generación de una nueva batería de políticas públicas y programas que atendieran algunas de las demandas planteadas por este grupo. En el 2003 fue promulgada la Ley de la Comisión Nacional para el Desarrollo de los Pueblos Indígenas (Diario Oficial de la Federación, 2003), que marcaba una transformación sustancial, aunque no estaba exenta de críticas y señalamientos, pues en términos de nuestro análisis representaba la transformación de los indígenas de un grupo dependiente de las políticas públicas a beneficiarios. Asimismo, en esta ley, el Estado mexicano postulaba el desarrollo de las comunidades indígenas como uno de sus objetivos. La cultura mestiza tenía ahora el mismo estatus que la de los pueblos indígenas.

\section{El Estado mexicano monopoliza la historia: las zonas arqueológicas.}

La construcción de las áreas de control de los Estados modernos no es un acto único y originario. Por el contrario, se trata de un proceso continuo que presenta áreas de avance y retroceso tanto por las diferencias de intereses entre los distintos agentes sociales involucrados como por el mismo desarrollo de los medios estatales para lograr dicho control. El proyecto estatal mexicano por lograr un monopolio en el control sobre el patrimonio histórico (material e inmaterial), ha logrado una paulatina expansión, sin embargo, inició con una serie de limitaciones que existían al término de la Revolución Mexicana; en otras palabras, el poder estatal 
mexicano sobre la cultura inició escaso y fragmentado en la década de los veinte y treinta del siglo pasado (Agudo y Estrada, 2014).

Un claro ejemplo de esta debilidad institucional del Estado mexicano para lograr la regulación sobre el patrimonio es la ley sobre Protección y Conservación de Monumentos Arqueológicos e Históricos, Poblaciones Típicas y Lugares de Belleza Natural, publicada el 19 de enero de 1934 (Diario Oficial de la Federación, 1934).' Esta ley generaba un marco general para el manejo de zonas o bienes considerados como patrimonio nacional. Sin embargo, su aplicación estaba limitada por el artículo 73 de la Constitución de 1917, fracción XXV, en un inicio, la nueva ley sobre Conservación de monumentos sólo tenía validez jurídica dentro del Distrito Federal y aquellos terrenos de control federal (Olivé, 1980).

El 5 de junio de 1972 entró en vigor la Ley Federal Sobre Monumentos y Zonas Arqueológicas, Artísticos e Históricos. Esta ley otorgaba al Estado mexicano la regulación en las tareas de "...investigación, protección, conservación, restauración y recuperación de los monumentos arqueológicos, artísticos e históricos y de las zonas de monumentos (Congreso de la Unión, 1986). Esta disposición permitió al Estado la formación de sujetos, definiendo a las dependencias que serían las responsables de su ejecución: El presidente de la República, El Secretario de Educación Pública, el Secretario de Patrimonio Nacional, El Instituto Nacional de Antropología e Historia y el Instituto Nacional de Bellas Artes.

El Estado mexicano generó un monopolio respecto con la construcción del patrimonio en diversos aspectos: a) declaración de monumentos arqueológicos, artísticos, históricos y zonas de monumentos (art. 5); $b$ ) el uso que los particulares puedan darles a los monumentos que se encuentran dentro de su propiedad (art. 6); c) permisos para la restauración y conservar los monumentos; $d$ ) comercio, reproducción y exportación de monumentos históricos (art. 15 -17); e) expertos (profetas del pasado) certificación, manejo, restauración, transporte, catalogar, etcétera de los monumentos históricos (art. 29- 34bis) (Congreso de la Unión, 1986).

La ley de 1972 construye la forma de racionalidad con la que se operará la protección y manejo de monumentos y zonas arqueológicas otorgando, en primer lugar, una dirección centralizada a las relaciones: la federación centraliza el control sobre las decisiones en esta materia. Las entidades estatales y municipales tienen un papel subordinado a las disposiciones federales. Por ejemplo, en el artículo 12 de esta Ley, los municipios tienen un papel auxiliar en casos de urgencia para lograr la suspensión provisional

1 Sobre la fecha de publicación existe un diferendo con el texto de Olive (1980) que la ubica el 30 de enero de 1930. 
de obras que pudieran generar un daño o alteración a bienes declarados monumentos (Congreso de la Unión, 1986:3).

El control estatal sobre la certificación de las personas que pueden declarar, clasificar manejar los monumentos históricos ((art. 29- 34bis) construye las formas características de visibilidad y maneras de percibir, pues le otorga al Estado el monopolio exclusivo de decir qué será un monumento histórico y que no lo será. En el artículo 28 se definen qué son los monumentos arqueológicos: “...los bienes muebles e inmuebles, productos de culturas anteriores al establecimiento de la hispánica en el territorio nacional, así como los restos humanos, de la flora y fauna, relacionados con esas culturas..." (Congreso de la Unión, 1986:5).

Un ejemplo de lo anterior está en la relación de las dependencias federales con las comunidades indígenas. A pesar del creciente papel que desempeñan los indígenas en la definición y uso de los símbolos y lugares relacionados al pasado prehispánico de México, el Estado mexicano continúa ejerciendo el monopolio sobre la definición cultural de la historia, los objetos, personajes y lugares que pueden ser considerados como zonas arqueológicas, turísticas o, partes de la historia oficial. (Johnson, 2014). Un ejemplo de ello es la investigación de Anne Johnson sobre la disputa que ha existido entre el pueblo de Ixcateopan, Guerrero y el Estado mexicano por reconocer como legítimas los huesos que reposan en la Iglesia de la Asunción como los pertenecientes al emperador mexica Cuauhtémoc. El gobierno mexicano ha nombrado tres comisiones distintas de expertos $(1949,1950,1976)$ que sistemáticamente han descartado la autenticidad de los huesos o los documentos que corroboran la versión de que ahí fueron depositados los restos mortales del último emperador azteca. Por su parte, la población local mantiene su creencia en la autenticidad de los restos, pero no sólo ellos, una creciente cantidad de turistas nacionales y extranjeros visitan el lugar. Las autoridades del Estado de Guerrero y del municipio han mostrado su reconocimiento implícito a la postura local mediante su presencia en las celebraciones por el "descubrimiento" de los huesos de Cuauhtémoc (Johnson, 2014).

La Ley Federal Sobre Monumentos y Zonas Arqueológicos, Artísticos e Históricos también crea formas de producir el conocimiento al generar el Registro Público de Monumentos y Zonas Arqueológicos e Históricos (Instituto Nacional de Antropología e Historia) y el Registro Público de Monumentos y Zonas Artísticos (Instituto Nacional de Bellas Artes y Literatura). Ambos están encargados de llevar el registro de todos los monumentos, estén este bajo control federal, estatal, municipal, personas físicas o morales privadas. 


\section{EI INAH.}

Como dijimos antes, los Estados tienen como objetivo central la producción de la verdad. Uno de los mecanismos para lograrlo son las formas de normalización, haciendo con ello referencia a los procedimientos estatales utilizados para enfrentar y conocer los fenómenos, el lograr determinar cuál es su funcionamiento regular normal, o bien, los diferentes grados con los que se presenta. En el caso que nos interesa analizar, el Estado mexicano creó una dependencia que específicamente se dedicaría a formar la verdad sobre su pasado: el Instituto Nacional de Antropología e Historia INAH. El decreto oficial de su creación fue publicado en el Diario Oficial de la federación el 3 de febrero de 1939.

En tanto política pública, la creación del INAH tenía una función regulatoria y redistributiva (Lowi, 1992); en el primer caso esta institución buscaría generar las reglas generales que delimitaran la actuación del gobierno y los demás actores sociales en lo relacionado con la exploración, vigilancia, conservación, restauración, de las zonas arqueológicas, así como la investigación y publicación científicas y artísticas relacionadas a la antropología, etnografía e historia de México (Diario Oficial de la Federación. 1939). En la segunda función, el INAH buscaría volver públicos bienes culturales de México, lo que significaría garantizar el acceso a los mismos a una amplia franja de la población que hasta este momento no los conocían o podían accederlos.

El INAH es la herramienta del Estado mexicano para construir el monopolio de las formas características de visibilidad y maneras de percibir y decidir cuáles serían las zonas, objetos y en general expresiones culturales que serán consideradas como parte del patrimonio. La clasificación de las zonas arqueológicas significa una selección de espacios y el descarte de otros. Con ello, esta dependencia produce formas de racionalidad mediante las cuales el Estado mexicano genera un conjunto de formas de comportamiento y de dirección de los comportamientos respecto con los lugares, objetos y prácticas que serán consideradas dentro de un binomio inicial: (bienes) patrimoniales/ no patrimoniales.

Para producir una forma específica y única de racionalizar los bienes patrimoniales el Estado mexicano por medio del INAH desarrolló tres acciones paralelas. Primera unificar en esta dependencia todas las agencias relacionadas a los que serían considerados bienes patrimoniales; fueron incorporados al INAH el Museo Nacional de Arqueología, Historia y Etnografía, la Dirección de Monumentos Prehispánicos y la Dirección de Monumentos Coloniales, la integración de la Escuela Nacional de Antropología (1940), la firma de convenios con los Estados para la 
formación de institutos regionales mixtos y la reorganización de distintos museos en todo el país en 1954 (Yáñez, 2006).

Segundo, el Estado mexicano produjo las directrices de acción que le permitieran construir una forma de acción unificada y permanente. Como lo señala Yáñez desde su formación el INAH institucionalizó una política cultural basada en tres ejes: 1) El nacionalismo, la soberanía y la identidad nacional como bases para el plan cultural estatal; 2) convertir la preservación de bienes considerados como patrimoniales como una política estatal continua e importante; 3) vincular la educación pública con la cultura nacional y el patrimonio cultural. Tercero, el INAH desarrolló una serie de oficinas técnicas encabezadas por sujetos con formaciones muy específicas, sus propios profetas éticos, que serían los únicos autorizados en definir lo patrimonial de un bien; Dirección de Prehistoria (1952), Dirección de Investigaciones Antropológicas (1954), Departamento de Acción Educativa (1953), Departamento de Promoción y Difusión (1965), que cambiaría después a Publicaciones e Investigaciones Históricas, Antropología Física y Lingüística (1970), Departamento de Proyectos Especiales (1979), Centro de Investigaciones Superiores (1973) (Yáñez, 2006).

El 28 de abril de 1972 fue promulgada la Ley Federal Sobre Monumentos y Zonas Arqueológicas, Artísticos e Históricos. Para el Estado mexicano esto resultó un paso fundamental en las formas de producir el conocimiento, ya que este documento contenía una conceptualización sobre qué sería considerado patrimonio y refrendando el rol central del Estado y sus instituciones para el manejo y conservación de estos bienes (Yáñez, 2006).

\section{Conclusiones.}

Como hemos visto, las políticas públicas son uno de los mecanismos estatales para promover que una determinada visión sobre las relaciones entre los grupos sociales, la definición de los problemas públicos y el uso de los recursos sean reconocidas por el resto de los grupos sociales como naturales, es decir como la realidad. Este proceso no es un acto estatal unilateral, se produce dentro de un entorno de interacciones donde existen fenómenos y actores que tienen capacidades para influir en ello. Esto último lo ilustra el caso de Ixcateopan, Guerrero donde existe una disputa por el reconocimiento de los huesos como pertenecientes al emperador mexica Cuauhtémoc.

El Estado constituye un ejercicio de poder mediante el cual busca intervenir sobre el campo de acción real o posible de los ciudadanos que habitan en su territorio. El poder puede ser definido como la posibilidad de ampliar o de restringir el campo de acción de los otros quienes se reconocen como actuantes y responsables, capaces de actuar, y sobre 
todo de responder (Foucault, 1999; Dean, 1999). Como vimos, una de las características centrales de los Estados modernos es su incesante búsqueda por conducir la conducta de todos los actores que se encuentran dentro de su territorio (Foucault, 2004). Las políticas públicas son los mecanismos concretos por medio de los cuales los Estados ejercen este control directo e indirecto sobre las conductas.

Foucault propuso el concepto de gubernamentalidad para explicar los mecanismos concretos mediante los cuales los Estados ejercen control sobre las conductas La gubernamentalidad hace referencia a un conjunto de procedimientos, instituciones, creencias e identidades por medio de los cuales se gobierna la conducta de una población (Foucautl, 1991). De tal forma que para el análisis de la intencionalidad estatal en las políticas públicas resultan centrales las capacidades, -los recursos organizativos, monetarios, conocimientos, procedimientos legales-, que tienen las estructuras estatales concretas para poder influir en la reconfiguración de un fenómeno específico como el turismo en la búsqueda por lograr el bienestar de la población. El problema de los recursos al analizar la intencionalidad toma importancia si consideramos que los Estados pueden tener un fuerte interés por transformar el curso de acción de un grupo o los procesos sociales que se desarrollan dentro de un lugar determinado, pero puede carecer de los medios para lograrlo. En el caso de los Estados Latinoamericanos no ha sido hasta fechas relativamente recientes en que han desarrollado los mecanismos y contado con los medios necesarios para influir de una manera decisiva en el desarrollo del turismo dentro de sus propios territorios.

La gestión social de las zonas arqueológicas no puede ser entendida simplemente como un acto burocrático y técnico. Esta es un área de construcción de la realidad social que ha buscado ser sistemáticamente monopolizada por los Estados nacionales. Para lograr este objetivo los Estados han desarrollado un conjunto de mecanismos y funcionarios que controlan tanto la definición sobre qué será considerado un monumento arqueológico, quién será el encargado de su clasificación, manejo, venta o uso. En otras palabras, el Estado (mexicano) se abroga la capacidad para definir cuál es nuestro pasado y cómo debe ser correctamente utilizado.

Entender la gestión como una parte de la construcción de un Estado, nos permite analizarla como un proceso inacabado y como parte de metas e ideologías sobre la conformación de la identidad general. La idea sobre el pasado mexicano ha sido transformada por los intereses y los funcionarios estatales encargados de su definición y conservación. 


\section{Referencias.}

Agudo Sanchíz Alejandro y Estrada Saavedra Marco (2014). Formas reales de la dominación del Estado. Perspectivas interdisciplinarias del poder y la política. México: El Colegio de México.

Anderson Benedict ([1993] 1983). Comunidades imaginarias. Reflexiones sobre el origen y la difusión del nacionalismo. México: Fondo de Cultura Económica.

Bonfil Batalla Guillermo. 1990. México profundo. Una civilización negada. Editorial Grijalvo. México.

Bourdieu Pierre (2014). Sobre el Estado. Cursos en el Collège de France (19891992). España: Anagrama. Colección argumentos.

Cancian Frank (1976). Economía y prestigio en una comunidad maya. El sistema religioso de cargos en Zincacantan. México: Instituto Nacional Indigenista.

Cárdenas Demay Almandina y Arellano Martínez Alejandra. 2004. “Hacia la definición de una política del lenguaje explícita en México". En Joan Argenter and McKenna Brown. Endangered Languages and Linguistic Rights. The Fundation for Endangered Lenguages. United Kingdom: 67-72.

Castells, Manuel. (2009). La era de la información. Economía sociedad y cultura. El poder de la identidad. Vol. II. México: Siglo Veintiuno Editores.

Congreso de la Unión (1986). Ley Federal Sobre Monumentos y Zonas Arqueológicos, Artísticos e Históricos. Versión Electrónica. (9 de octubre de 2017). http://www.wipo.int/wipolex/es/text.jsp?file_id=220855

Cosío Villegas Daniel, Bernal Ignacio, Moreno Toscano Alejandra, Gonzáles Luis y Blanquel Eduardo. (1974). Historia mínima de México. México: El Colegio de México.

Diario Oficial de la Federación (1934). Gobierno Federal Mexicano. Tomo LXXXII. Vol. 16. (19 de enero de 1934). http://www.dof.gob.mx/index.php?year=1934\&month=01\&day=19

Diario Oficial de la Federación (1939). Gobierno Federal Mexicano. Tomo CXII. Núm. 29. (viernes 3 de febrero de 1939). http://www.dof.gob.mx/index. php?year $=1939 \&$ month $=02 \&$ day $=03$

Diario Oficial de la Federación. 2003. Gobierno Federal Mexicano. Tomo DXCVI. No. 13. (Miércoles 21 de mayo). http://www.dof.gob.mx/nota_to_imagen_ fs.php?codnota $=695791 \&$ fecha $=21 / 05 / 2003 \&$ cod_diario $=28264$

Díaz-Andreu Margarita. 2014. “Turismo y Arqueología. Una mirada histórica a una relación silenciada". Anales de Antropología. 48(II): 9-39.

Dean, Mitchell (1999). Governamentality. Power and Rule in Modern Society. London: Sage Publications.

Durkheim Émile ([1912] 2013). Las formas elementales de la vida religiosa. El sistema totémico en Australia (y otros escritos sobre religión y conocimiento). México: Fondo de Cultura Económica. 
Elias Norbet (2016). El proceso de la civilización. Investigaciones sociogenéticas y psicogenéticas. México: Fondo de Cultura Económica.

Etzioni Amitai (1967). "Mixed-Scanning: a Third Approach to Decision-Making". Administration Review. No. XXVII: 385-392.

Foucault, Michelle (1991). "Governamentality" en Graham Bruchell, Colin Gordon and Peter Miller (eds.), The Foucault Effect: Studies in Governamentality, USA:Chicago University Press: 87- 104.

Foucault, Michel (2000). Defender la sociedad, Fondo de Cultura Económica. Argentina: Buenos Aires.

Foucault, Michel (2004). Seguridad, territorio y población. Fondo de Cultura Económica. Argentina: Buenos Aires.

Giddens Anthony. 1985. The Nation-state and Violence. University of California Press. Harris Marvin (1964). Patterns of race in the Americas. New York: Walker and Company. Johnson Anne W. (2014). “El poder de los huesos: peregrinaje e identidad en Ixcateopan de Cuauhtémoc, Guerrero". Anales de Antropología. 48(II): 119- 149.

Iturralde Diego. 1991 "Los pueblos indios como nuevos sujetos sociales en los Estados latinoamericanos" Nueva Antropología XI(39): 27-40.

López Villafañe Víctor. 1986. La formación del Sistema político mexicano. Siglo Veintiuno editores. México.

Lowi Theodore J. (1992). "Políticas públicas, estudios de caso y teoría política". En Luis F. Aguilar Villanueva (edición). La hechura de las políticas. México: Miguel Ángel Porrúa Grupo Editorial: 77- 118.

Mallon Florencia. 1994. "Reflections on the Ruins: Everyday Forms of State Formation in Nineteenth- Century Mexico. In Gilbert M. Joseph and Daniel Nuget. Everyday Forms of State Formation. Duke University Press. United State of America: 69-106.

Marx Karl ([1875] 2004). Crítica del programa de Gotha. España: El Cid Editor.

Olivé, Julio César (1980). “Reseña histórica del pensamiento sobre arqueología” en Arqueología y Derecho, Instituto de Investigaciones Antropológicas. México: UNAM.

Oseguera Andrés. 2008. “De ritos y antropólogos. Perspectivas teóricas sobre el ritual indígena en la antropología realizada en México". Cuicuilco. Vol. 15. Núm. 42. (enero-abril): 97-118.

Redfield, Robert. 1930 Tepoztlán, a Mexican Village: A Study of Folk Life. Chicago: University of Chicago Press.

Rockwell Elsie. 1994. "Schools of the Revolution: Enacting and Contesting State Forms in Tlaxcala, 1910-1930". In Gilbert M. Joseph and Daniel Nuget. Everyday Forms of State Formation. Duke University Press. United State of America: 170- 208.

Schneider Anne and Ingram Helene, (1993)," Social Construction of Target Population: Implications for Politics and Policy, The American Political Review, Vol. 8, No. 2 (jun) pp. 334-347. 
Sofus Lumholtz Carl (1945). El México desconocido. Cinco años de exploración entre las tribus de la Sierra Madre Occidental; en la tierra caliente de Tepic y Jalisco, y entre los Tarascos de Michoacán. New York: Charles Scribner's Sons.

Weber Max. ([1919] 1984). El político y el científico. México: Premia.

Weber, Max (2009). La política como vocación. España: Alianza Editorial.

Wendell Bennett y Robert Zingg (1935) The Tarahumara, an Indian tribe of northern Mexico. Chicago: University of Chicago Press.

Yáñez Reyes, Sergio (2006). “El Instituto Nacional de Antropología e Historia: antecedentes, trayectoria y cambios a partir de la creación del CONACULTA". Cuicuilco, vol. 13, núm. 38, septiembre-diciembre: 47-72.

Van Zantwijk Rudolf (1974). Los servidores de los santos. La identidad social y cultural de una comunidad tarasca. México: Consejo Nacional para la Cultura y las Artes.

Villalobos Acosta, Cesar. 2011. Archaeology in Circulation: Nationalism and Tourism in Post-Revolutionary Mexican Coins, Notes, Stamps and Guidebooks. Doctoral thesis, Durham University. Available at Durham E-Theses Online: http://etheses.dur.ac.uk/3211/

Velázquez García Mario (2016). “Recursos del Estado mexicano contra los movimientos sociales: las distintas manos de leviatán". Estudios Sociológicos. Vol. XXXIV. Núm. 101 (mayo-agosto): 247-272.

Velázquez Guzmán, María Guadalupe (1982). “Afectaciones petroleras en Tabasco: el movimiento del Pacto Ribereño", Revista Mexicana de Sociología, vol. 44, núm. 1, pp. 167-187. 